\title{
ARTIGO ESPECIAL
}

\section{BIODISPONIBILIDADE DE MINERAIS}

\author{
MINERAL BIOVAILABILITY
}

Silvia M. Francisc ato Cozzolino ${ }^{1}$

RESUMO

A importância da determinação da biodisponibilidade de minerais em dietas está centralizada no estabelecimento das recomendações de ingestão destes elementos em função das necessidades dos indivíduos. Assim, os estudos de biodisponibilidade de nutrientes devem ser específicos para cada país, tendo em vista a grande diversidade de dietas e de indivíduos. Nesta abordagem, procurou-se discutir as metodologias disponíveis para avaliação da biodisponibilidade de minerais e os fatores que podem interferir nesta medida, visando motivar jovens pesquisadores a se interessarem por esta área de pesquisa que promete grandes descobertas.

Termos de indexação: biodisponibilidade de minerais, dieta.

\begin{abstract}
The importance of mineral bioavailability determination in diets is focused on the establishment of these minerals intake recommendations, considering the individual necessities. So, researche of nutrients bioavailability should be specific to each country, taking into account the numerous types of diets and individuals. The purpose of this article is to discuss the available methodologies for mineral bioavailability assessment and the variables that interfere with this evaluation, trying to make young researcher interested in this area which is quite promising in new discoveries.
\end{abstract}

Index terms: mineral bioavailability, diet.

\section{INTRODUÇÃO}

O estudo dos minerais teve um grande avanço a partir da década de 70, com o desenvolvimento de técnicas analíticas mais sensíveis e precisas, que permitiram não apenas a quantificação de elementos encontrados como traços em alimentos e fluídos biológicos, mas também desvendar alguns dos

\footnotetext{
(1) Professora Associada da Faculdade de Ciências Farmacêuticas da Universidade de São Paulo, Av. Lineu Prestes, 580, 05508-900, São Paulo, SP,Fax (011) 815-4410; E-mail: smfcozzo@usp.br.
} 
mecanismos através dos quais estes exercem suas funções no organismo.

Portanto, daquela época até os dias atuais, uma preocupação dos pesquisadores tem sido quantificar estes elementos nas dietas de grupos da população, visando avaliar a ingestão destes micronutrientes e correlacionar estes achados com o estado nutricional dos indivíduos. O objetivo desses estudos é a determinação mais precisa das necessidades do organismo e o estabelecimento das recomendações de ingestão alimentar por meio dos conhecimentos da biodisponibilidade nas dietas. Entretanto, para atingir estes objetivos, muitos problemas ainda precisam ser resolvidos.

\subsection{Ava lia ção do c onsumo a limenta r}

Existem muitas dúvidas quanto a precisão da coleta de dados sobre consumo alimentar. Dos vários métodos propostos todos apresentam algum tipo de erro, e em todo mundo estudos estão sendo desenvolvidos visando pelo menos minimizá-los (GIBSON, 1990; BUZZARD \& SIEVERT, 1994; HANKIN \& WILKENS, 1994; KOHLMEIER, 1994; GUENTHER et al., 1997).

Dentre os mais aceitos atualmente para a obtenção de dados de consumo de micronutrientes se encontra o método da análise em duplicata da dieta consumida. Entretanto, é um método trabalhoso e difícil de ser realizado com grandes grupos da população, pois se baseia na análise química da duplicata da dieta consumida pelo indivíduo. Para uma maior representatividade, as dietas devem ser coletadas pelo menos por três dias (dois dias durante a semana e um no final de semana) e, num estudo mais completo, nas diferentes estações do ano.

Com relação ao registro alimentar, também realizado durante três dias da mesma forma proposta anteriormente, necessita da conscientização do indivíduo para o estudo. Sua precisão dependerá do rigor no registro dos alimentos ingeridos e das medidas adotadas para expressar as quantidades. O método engloba ainda as divergências de conteúdo de nutrientes devido à utilização de tabelas de composição de alimentos. Para minimizar estes erros, poderia ser recomendada a reprodução da dieta com os alimentos da região e posteriormente sua análise química.

O método recordatório de 24 horas, muito utilizado por ser de fácil aplicação, validado para determinados grupos da população, não é recomendado para idosos. Apresenta maiores limitações que os dois anteriores e implica no aumento do tamanho da amostra.

Outros métodos que empregam determinações bioquímicas para validação dos dados de inquéritos alimentares também tem sido recomendados (JACQUES et al., 1993). Portanto, ao se avaliar os resultados de uma pesquisa, deve-se sempre analisar a metodologia utilizada e dispensar o devido cuidado na sua interpretação.

\subsection{Ava liação do esta do nutric ional}

É grande a dificuldade em se estabelecer o estado nutricional dos indivíduos de uma população em relação aos minerais, pois ainda não existem parâmetros bem definidos para a maioria dos elementos. Em relação ao ferro pode-se considerar que os conhecimentos estão mais avançados, enquanto que para outros como zinco, selênio, cobre, cálcio e magnésio, ainda há necessidade de maiores estudos (GIBSON, 1990; BURTIS \& ASHWOOD, 1994).

\subsection{Necessida dese recomendações}

Finalmente, o estabelecimento das necessidades de nutrientes e das recomendações de ingestão alimentar , tem motivado discussões infindáveis na comunidade científica, tanto para revisão das Recommended Dietary Allowances (RDA), como para estabelecimento das Recommended Dietary Intake (RDI) (BEATON, 1996; JOHNSON, 1996; LUKASKI \& PENLAND, 1996; O’DELL, 1996; TUCKER, 1996; HATHCOCK, 1997), uma vez que não apenas se pretende estabelecer o mínimo necessário, mas também o nível ideal para a prevenção de doenças consideradas crônicas.

\section{DEFINIÇÃO DE BIODISPONIBILIDADE}

O termo biodisponibilidade foi proposto inicialmente para a área farmacológica, visando estabelecer a proporção em que determinada droga intacta alcança a circulação e a razão na qual isso ocorre.

Na década de 80, partindo do princípio que a simples presença do nutriente na dieta não garante sua 
utilização pelo organismo, passou-se a utilizar este termo para indicar a proporção do nutriente que é realmente utilizada pelo organismo (SOUTHGATE et al., 1989). Esta definição, aceita preferencialmente como um conceito, persistiu até pouco tempo, entretanto, em 1997, no Congresso debiodisponibilidade realizado em Wageningen na Holanda, foi proposta uma redefinição, ou seja: "Biodisponibilidade é a fração de qualquer nutriente ingerido que tem o potencial para suprir demandas fisiológicas em tecidos alvos", e que provávelmente passará a vigorar a partir desta data.

\section{ETAPAS A SEGUIR NOS ESTUDOS DE BIODIS- PONIBIUDADE}

Com o conhecimento da biodisponibilidade dos minerais, considerando os fatores da dieta e do indivíduo, as recomendações destes nutrientes poderão ser estabelecidas com maior precisão e desta forma elaborar os guias de alimentação específicos para cada país.

Quanto aos estudos de biodisponibilidade, também foi sugerido neste último Congresso a utilização do termo: SLAMANGHI, que foi proposto para o estudo de carotenóides e considerou-se que poderia ser utilizado também para os demais nutrientes. O termo lembra os aspectos que devem ser considerados nos estudos de biodisponibilidade, e cada letra tem seu significado: $\mathrm{S}$ = Species (especiação do nutriente); $\mathrm{L}=$ Linkage (ligação molecular); $\mathrm{A}=$ Amount in the diet (quantidade na dieta); $\mathrm{M}=$ Matrix (matrix onde o nutriente está incorporado); $\mathrm{A}=$ Attenuators of absorption and bioconversion (atenuadores da absorção e bioconversão); $\mathrm{N}=$ Nutrient Status (estado nutricional do indivíduo); G = Genetic factors (fatores genéticos), $\mathrm{H}=$ Host related factors (fatores relacionados ao indivíduo); e I = Interactions (interações).

Portanto, neste artigo, estes aspectos serão abordados ao discorrermos sobre biodisponibilidade de minerais e suas interações no organismo.

\section{METODOLOGIAS PARA DETERMINAÇÃO DA BIODISPONIBILIDADE}

Os métodos utilizados para determinação da biodisponibilidade de nutrientes, procuram quantificar a porcentagem do nutriente ingerido que tem o potencial de suprir as demandas fisiológicas do organismo. Os trabalhos iniciais nesta área se baseavam em estudos de balanço onde se verificava quanto do nutriente ingerido era eliminado pelas vias de excreção e se considerava a fração resultante como a biodisponibilidade. Entretanto, estes estudos não consideravam a fração endógena do nutriente e não era possível medir com precisão a quanto ela correspondia.

Os métodos de depleção/repleção vieram a seguir, e como o nome indica, são baseados na depleção do nutriente em estudo, até o aparecimento dos sinais bioquímicose clínicos da deficiência, seguidos da repleção com fontes do nutriente presentes em alimentos ou compostos isolados, onde se mede a eficiência da utilização do nutriente fornecido, ou seja sua biodisponibilidade. Estes estudos são realizados tanto em animais de laboratório como em humanos voluntários. A biodisponibilidade se determina, a partir da quantidade do nutriente ingerido que foi absorvido, correlacionando estes resultados com a concentração do elemento nos tecidos e fluídos biológicos e/ou compostos dependentes do elemento (ex. enzimas, fatores de transcrição).

Paralelamente, os métodos utilizando isótopos radioativos começaram a se desenvolver nesta área e a partir de então foi possível a obtenção de dados mais reais de biodisponibilidade de minerais, pois se podia marcar um elemento químico radioativamente e posteriormente analisá-lo nos diversos materiais biológicos. Esta metodologia pode ser utilizada em animais, bem como no ser humano, porém neste último caso excluindo crianças e mulheres na idade fértil.

As técnicas "in vitro" para estudos de absorção de minerais, também tem sido validadas e aperfeiçoadas por meio da utilização de cultura de células. Espera-se que esta metodologia possa ser utilizada como passo inicial para o conhecimento da biodisponibilidade, pois, embora se considere que a absorção seja a etapa mais importante neste processo, isoladamente, não tem o mesmo significado de biodisponibilidade(GLAHN \& VANCAMPEN, 1997; GODDARD et al., 1997).

Métodos mais avançados, utilizando isótopos estáveis, estão sendo cada vez mais empregados para avaliação da biodisponibilidade de minerais, tendo ocorrido um grande desenvolvimento a partir da década de 80. Dentre as suas vantagens está a possibilidade 
de estudar grupos de risco como crianças, gestantes e lactantes. A sua principal desvantagem é o custo, pois a obtenção dos isótopos enriquecidos é muito difícil e cara, além do que a metodologia de análise necessita de equipamentos sofisticados. MELLON \& SANDSTRÖM (1996), editaram um livro orientando a utilização desta metodologia, que servirá de guia aos interessados em realizar trabalhos nesta área.

Na escolha da metodologia para estudos desta natureza no Brasil, o pesquisador deve utilizar todos os recursos disponíveis no seu laboratório e procurar parceiros que o auxiliem em laboratórios melhor equipados, deve validar sua metodologia analítica utilizando materiais de referência certificados, e, principalmente, trabalhar com alimentos e dietas regionais, pois, em nosso país, há carência de informações sobre a biodisponibilidade de minerais.

\section{FATORES QUE INTERFEREM NA BIODISPO- NIBILIDADE DE MINERAIS}

Existe uma seqüência de eventos que pode interferirnas medidas de biodisponibilidade deminerais. Alguns destes fatores podem ser trabalhados no sentido positivo de se conseguir um melhor aproveitamento dos nutrientes. No caso dos minerais, entretanto, os fatores que se relacionam ao indivíduo são mais difíceis de serem controlados e poderemos obter respostas muito diferentes para o mesmo alimento, refeição ou dieta (REDDY \& COOK, 1997; HALLBERG et al., 1997). Discorreremos sobre estes eventos de acordo com a proposta SLAMANGHI.

\subsection{Especiação}

Comecemos pela ordem com a especiação, isto é, a fórmula química na qual o elemento se encontra no alimento ou dieta, que é o primeiro passo para determinarmos seu aproveitamento pelo organismo. Os minerais podem estar presentes nos alimentos na forma livre ou combinada, necessitando ou não de digestão para serem absorvidos. O estado de oxidação é também um fator importante.

\subsection{Ligação molecular}

Dependendo da estrutura atômica, ligação dos átomos, isto é ligações covalentes ou iônicas, por pontes de hidrogênio, grupos sulfídricos, etc., bem como dos modelos de ligação nas esferas de coordenação dos metais e dos ligantes e seus estados de oxidação, poderemos ter solubilidades diferentes em água, meio ácido ou básico, que poderão ter influênciana biodisponibilidade.

\subsection{Qua ntidade ingerida}

Em relação às quantidades ingeridas numa refeição ou dieta, podemos admitir que o organismo normal tenta manter sua homeostase e geralmente absorve mais quando suas reservas estão diminuidas e menos quando estão em condições adequadas ou de excesso. Por outro lado, o excesso de um nutriente pode interferir no aproveitamento de outro. Portanto, ao avaliarmos a biodisponibilidade de um mineral específico, se não forem verificadas as reservas do mesmo no organismo, as respostas poderão ser interpretadas de maneira equivocada.

\subsection{Matrix a limentar}

Na matrix alimentar podemos ter compostos que impedem a absorção de minerais. Podemos citar como exemplo o ferro na gema do ovo, que se encontra ligado à fosvitina, uma proteína que representa 7\% das proteínas da gema e tem uma alta capacidade ligante(LYNCH, 1997); no espinafre, o alto conteúdo de oxalato se liga ao ferro e ao cálcio diminuindo a biodisponibilidade destes nutrientes. Nos cereais, o fitato presente pode se ligar ao ferro, zinco, cálcio e magnésio diminuindo também a absorção, entretanto, deve-se ressaltar que existem diferentes formas de fitato, com características inibitórias diferenciadas (FAIRWEATHER-TAIT, 1996).

Nos alimentos ricos em fibra, os minerais podem estar presentes nestas frações e, portanto, não disponíveis para absorção. Porém, especificamente em relação a este aspecto, os resultados são muito conflitantes; muitos autores consideram que é o fitato presente nesta fração o principal responsável pela diminuição da biodisponibilidade, e este efeito é maior para o zinco. Atualmente, estudos estão sendo conduzidos nesta área e se verifica uma preocupação, principalmente ao se indicar $35 \mathrm{~g}$ de fibra alimentar por 
dia, visando a prevenção de doenças. Produtos da reação de Maillard, também podem complexar metais, principalmente $\mathrm{Zn}, \mathrm{Cu}, \mathrm{Mg}$ e $\mathrm{Ca}$ (O’BRIEN \& MORRISSEY, 1997).

Por outro lado, nas carnes, o ferro ligado à hemoglobina ou mioglobina, é melhor utilizado. É importante ressaltar que apenas $40 \%$ do ferro presente nas carnes é ferro heme, o restante também faz parte do ferro não heme.

\subsection{Atenua dores da a bso rçã o e bioc onversã o}

A presença nas dietas de ligantes antagônicos ou facilitadores, pode ter influência na absorção e bioconversão para a forma ativa, ou seja, para a forma funcional do elemento. Podemos citar como exemplo os fitatos, taninos e oxalatos que podem diminuir a absorção e por outro lado, ácidos orgânicos e alguns aminoácidos, que podem facilitar a absorção e bioconversão.

\subsection{Esta do nutric ional}

A dificuldade em se conhecer o estado nutricional do indivíduo no início de um estudo de biodisponibilidade é um fator de erro na determinação desta. Muitos estudos costumam avaliar previamente a capacidade de absorção de uma dose considerada de referência, por exemplo no caso do ferro, a absorção de uma dose de sulfato ferroso com ascorbato, isto para determinar as diferenças iniciais entre os indivíduos selecionados para o estudo (HALLBERG, 1980). Esta avaliação também tem sido realizada para outros minerais, verificando-se a eficiência da absorção de um elemento presente num alimento teste, comparada com uma dose de referência padrão. O resultado será a razão entre os dois (FAIRWEATHER-TAIT, 1996).

\subsection{Fatores genétic os}

Determinados fatores genéticos são responsáveis por alterações na absorção de alguns minerais. Podemos citar, como exemplo, indivíduos portadores de hemocromatose, onde ocorre aumento de absorção do ferro. Atualmente tem-se procurado estudar estes pacientes, visando desvendar os mecanismos de regulação da absorção deste mineral (BEUTLER, 1997). Na acrodermatite enteropática, desordem genética que leva à deficiência de zinco, existe dificuldade na absorção deste mineral (AGGETT, 1989) e no caso da síndrome de Menkes, ocorre um defeito de transporte de cobre levando a deficiência do mesmo (LINDER \& HAZEGH-AZAM, 1996).

\subsection{Fatores relaciona dos a os indivíduos}

Os fatores relacionados ao indivíduo dizem respeito ao sexo, idade e etapa do desenvolvimento fisiológico. Uma criança em desenvolvimento tem necessidades aumentadas de minerais para seu crescimento, e, portanto, terá uma capacidade de absorção e utilização aumentada, o mesmo acontecendo com gestantes e lactantes. Entretanto tal fato não ocorrerá com os idosos, que poderão ter deficiências por exemplo, na secreção de ácido clorídrico, importante para manutenção do pH ácido para solubilização dos minerais. Doenças também podem modificar a utilização dos nutrientes, assim como os medicamentos, que podem interagir com os minerais, na maioria das vezes diminuindo sua biodisponibilidade.

\subsection{Interações}

Com o conhecimento das interações entre nutrientes, e a influência dos demais componentes dos alimentos numa refeição ou dieta, podem-se controlar algumas das variáveis e desta forma interferir na utilização dos nutrientes. Portanto, daremos maior ênfase a estes aspectos da biodisponibilidade nos parágrafos seguintes.

\subsubsection{Intera ções minera is versus minera is}

As interações minerais versus minerais podem ser diretas quando estes competem pelo mesmo sítio de absorção, por possuirem propriedades físicas e químicas semelhantes, e portanto o excesso de um prejudicará a utilização do outro, e, indireta, quando o mineral depende de outro para ser transformado para sua forma ativa, portanto a deficiência deste acarretará num prejuizo de função (COUZY et al.,1993). 
É importante também salientarmos que quando os minerais possuem propriedades semelhantes eles podem interagir não apenas no lúmem intestinal, mas também dentro do enterócito e mesmo a nível de transporte no sangue e para os tecidos.

\subsubsection{Interação Fe versus Zn}

Esta interação pode ocorrer tanto com a aumento do ferro interferindo na biodisponibilidade de zinco como em sentido contrário. Esta é uma interação direta. Experimento com ratos realizados em nosso laboratório, demonstrou que o aumento da concentração de ferro na dieta (4 vezes o teor encontrado), interferiu negativamente no aproveitamento do zinco (PEDROSA \& COZZOLINO, 1993). SANDSTRÖM et al. (1985), não reportaram inibição da absorção de zinco na razão molar de ferro para zinco de 2,5:1,0, quando ambos foram administrados com água, entretanto, quando a razão passou para 25:1 houve redução significativa do zinco. Portanto, parece que qualquer influência que o ferro possa exercer na absorção de zinco é muito menor na presença de ligantes de alimentos, que modificam a biodisponibilidade dos dois elementos, e, mesmo alimentos fortificados com ferro, parecem não ser prejudiciais ao zinco, a menos que a ingestão de zinco seja muito baixa. Assim, quando se pretende iniciar um programa de suplementação com ferro devido aos altos índices de anemia, não se deve discuidar do zinco no sentido de evitar esta interação. Por outro lado, o excesso de zinco também pode ter efeito na utilização do ferro. YARDICK et al. (1989), reportaram uma queda nos níveis de hematócrito e ferritina sérica de mulheres voluntárias de 25 a 40 anos, com suplementos de 50mg de zinco por dia.

\subsubsection{Interação Fe versus Ca}

Há muito tempo tem-se observado que dietas ricas em cálcio diminuem a biodisponibilidade do ferro (WHITING \& WOOD, 1997). Atualmente, este efeito está sendo mais pesquisado em função da recomendação dos suplementos de cálcio para prevenção da osteoporose. Em um trabalho recente, REDDY \& COOK (1997), estudando o efeito da ingestão de cálcio na absorção de ferro não heme de uma dieta completa, não verificaram efeito significativo na absorção de ferro, mas, pela diversidade dos resultados obtidos, e inclusive, nas reservas de ferro dos indivíduos, aconselharam cautela e recomendaram maiores investigações. De acordo com estes mesmos autores, existem evidencias convincentes que determinadas formas de suplementos de cálcio podem inibir a absorção de ferro não heme se ingeridos simultaneamente e o grau de inibição parece estar relacionado com a dose. Adições até 300mg, corresponderiam a um declínio de 50 a 60\% na absorção do ferro não heme. Estudo de GLEERUP et al. (1995), sobre a absorção de ferro de uma dieta completa associada a diferentes horários de ingestão de cálcio, concluiu que a absorção poderia aumentar de 1,32mg para 1,76mg de ferro diário (34\%), se a ingestão de cálcio se desse apenas no desjejum e na ceia noturna. Segundo COUZY et al. (1993), uma razão $\mathrm{Ca} / \mathrm{Fe}>150$, já foi documentada como de risco.

\subsubsection{Interação Ca versus Zn}

Esta interação tem um efeito mais pronunciado quando a dieta também é rica em fitatos (hexafosfato de mioinositol), pois na presença de cálcio o complexo Ca:fitato/Zn formado pode afetar adversamente o balanço de zinco no ser humano. A razão crítica, entretanto, ainda não foi determinada, acredita-se, que uma relação molar Ca: fitato/Zn $>200 \mathrm{mmol} /$ $1000 \mathrm{kcal}$ possa ocasionar problemas em dietas vegetarianas ou em dietas de populações de países em desenvolvimento, cuja ingestão de zinco é baixa associada a altos teores de fitato (FAIRWEATHERTAIT, 1996). Trabalho realizado por WOOD \& ZHENG (1997), também demonstra que a ingestão de 600mg de cálcio junto com a refeição diminui a absorção de zinco em 50\%, e concluíram que dietas com altos teores de cálcio podem aumentar as necessidades de zinco em humanos adultos.

\subsubsection{Interação $\mathrm{Zn}$ versus $\mathrm{Cu}$}

Esta interação tem sido bastante documentada, porque pode ser utilizada para tratamento de pacientes com doença de Wilson, onde o excesso de zinco diminue a absorção do cobre (BREWER et al., 1990). O mecanismo proposto para esta interação está baseado na observação de que o excesso de zinco leva ao aumento da síntese da metalotioneina, uma proteína, 
que tem como propriedade se ligar a minerais, protegendo o organismo dos possíveis efeitos tóxicos dos mesmos (COUSINS, 1994). Esta proteína tem uma afinidade maior por Cd, Cu e menor para o Zn, e seria um fator regulador da absorção de zinco ao nível do enterócito. Atualmente, tem-se proposto outras ações para esta proteína, que é altamente eficaz no sentido de impedir que quantidades consideradas tóxicas possam ser absorvidas. Pelo fato do cobre possuir maior afinidade pela metalotioneína, este ficaria retido no interior do enterócito, impedido de passar para a circulação e com a descamação das células entre 2 a 3 dias, seria excretado. Estudo apresentado por SANDSTEAD (1995), demonstrou que uma dose de $60 \mathrm{mg} /$ dia de zinco, causou depressão da superóxido dismutase dependente de Cu e Zn (EC 1.15.1.1). Esta interação também é importante ao se considerar, que a suplementação com ferro pode também recomendar uma suplementação de zinco e neste caso se houver deficiência de cobre, pode comprometer seu aproveitamento levando a deficiência. Ressalta-se que a deficiência de cobre pode causar diminuição da ceruloplasmina responsável pela transformação do $\mathrm{Fe}^{2+}$ para $\mathrm{Fe}^{3+}$ necessária para a síntese de hemoglobina, podendo levar a anemia.

\subsubsection{Interação Ca versus Mg}

Acredita-se que esta interação não tenha uma significância maior para o ser humano. Entretanto, com a recomendação da suplementação de cálcio numa dieta limítrofe ou baixa em magnésio, este efeito poderá ser relevante e deve ser mais pesquisado (COUZY et al., 1993).

\subsubsection{Interação $\mathrm{Zn}$ versus $\mathrm{Cd}$, Se versus $\mathrm{Hg}$}

Alguns metais pesados, como por exemplo o cádmio e o mercúrio, podem se complexar com minerais essenciais como zinco e selênio no trato gastro intestinal, e desta forma serem excretados. Este aspecto éimportante devido a diminuição da toxicidade, porém, a biodisponibilidade dos elementos essenciais é diminuída e pode ocasionar deficiências destes minerais em locais muito poluídos, quando associados com uma baixa ingestão destes elementos. Também tem-se observado que com a deficiência de cálcio, ferro, zinco e cobre na dieta ocorre maior absorção de chumbo.

\subsubsection{Interação Se versus lodo}

O selênio é importante para o metabolismo do iodo, pois para transformar a tiroxina (T4) em triiodotironina (T3) há necessidade da enzima selenodependente (deiodinase). Portanto, esta é uma interação indireta. São conhecidas três deiodinases que são dependentes de selênio (tipo I, II e III). Ultimamente, tem sido proposta autilização da atividade destas enzimas para estudar as interações entre selênio e iodo em culturas de tecidos de humanos. Em populações com deficiência de iodo e de selênio, estes dois elementos devem ser suplementados concomitantemente, uma vez que a suplementação somente com selênio poderia ser danosa, pois resultaria numa maior produção de T3, a partir do T4 residual, que promoveria uma inibição da liberação de TSH, piorando o quadro de hipotiroidismo(LEVANDER \& WHANGER, 1996).

\subsection{Minerais versus Vitaminas}

\subsubsection{Interação Fe versus Vitamina C}

É bastante conhecida a interação do ferro com a vitamina C (ácido ascórbico), facilitando a absorção do Fe não heme de uma refeição. Segundo LYNCH (1997), esta é uma interação direta porque independe do estado nutricional em relação a vitamina C. Porém, recentemente tem se demonstrado que a vitamina $\mathrm{C}$ também podeinfluenciar o transportee armazenamento do Fe no organismo. Indivíduos com deficiência de vitamina $\mathrm{C}$ tem defeito na liberação do Fe das células endoteliais. Observações de TOTH \& BRIDGES (1995), sugerem que o ácido ascórbico pode ser importante na modulação da síntese de ferritina e portanto no armazenamento de Fe. A influência da vitamina $C$ é mais pronunciada em refeições inibitórias, principalmente se estas contém altos teores de fitatos e polifenóis. O efeito é menor em refeições que contém carnes. Com a oxidação da vitamina provocada por preparação de alimentos a altas temperaturas há perda destas propriedades. O ácido ascórbico mantém o Fe na forma solúvel (se complexa ao Fe formando um quelado) biodisponível quando o $\mathrm{pH}$ do intestino aumenta (LINCH, 1997).

\subsubsection{Interação Cu versus Vitamina C}

O excesso de vitamina $\mathrm{C}$ pode prejudicar a absorção de cobre, havendo evidências de que este 
efeito ocorre pela redução do $\mathrm{Cu}^{2+}$ para $\mathrm{Cu}^{+}$, forma menos biodisponível. Acredita-se, que esta interação pode ser menos pronunciada em humanos que em animais (LÖNNERDAL, 1996). VAN DEN BERG \& BEYNEN (1992), avaliaram o efeito do ácido ascórbico na absorção de cobre e os efeitos pós absortivos no metabolismo deste mineral. Observaram redução da absorção, aumento da captação pelo fígado assim como da excreção biliar, utilizando ${ }^{64} \mathrm{Cu}$. Verificaram também, que o efeito era mais pronunciado nos animais deficientes em cobre. Estudos com humanos não tem demonstrado estes efeitos com doses de ácido ascórbico variando de 5 a 605mg/dia (LINDER \& HAZEGH-AZAM, 1996).

\subsubsection{Interação Fe versus Vitamina A}

Tem sido observada uma correlação direta entre retinol sérico e níveis de hemoglobina. Os estudos sugerem que a deficiência de vitamina A prejudica a mobilização de Fe das reservas e também a produção de células vermelhas, porém tem pouca influência na absorção de Fe (LYNCH, 1997).

\subsubsection{Interação Zn versus Vitamina A}

No caso do zinco, é amplamente conhecido que a proteína ligadora do retinol (RBP), responsável pelo transporte da vitamina A do fígado para os tecidos alvos é dependente de zinco. Portanto, um sinal clínico da deficiência do zinco é a cegueira noturna provocada pela dificuldade no transporte da vitamina A. Trabalho desenvolvido em nosso laboratório demonstrou este efeito em ratos depletados de zinco e de zinco e vitamina A, (YUYAMA \& COZZOLINO, 1995).

\subsubsection{Interação Se versus Vitamina E}

O selênio por meio da glutationa peroxidase (GSH Px), enzima dependente de selênio, age na redução de peróxidos orgânicos, incluindo peróxidos de ácidos graxos livres e outros lípides. A relação estreita entre selênio e vitamina $E$ tem base bioquímica. Vitamina $\mathrm{E}$ age minimizando o dano na membrana provocado pelos radicais livres; o selênio, por meio da glutationa peroxidase (GSH Px), age prevenindo o acúmulo de peróxido $(\mathrm{HOOH})$, que é fonte de radicais livres. Radicais hidroxila podem causar danos às membranas das células, bem como a outros componentes celulares. Assim, a necessidade de vitamina E é diminuída na presença de selênio (BRODY, 1994).

\subsection{Outras interações}

A literatura apresenta ainda outras interações com minerais entre si e com vitaminas, porém, como discutimos inicialmente, nosso objetivo não foi fazer uma revisão sobre o tema, mas sim trazer alguma contribuição nesta área. A interação do cálcio com a vitamina $\mathrm{D}$ não foi abordada por estar muito bem documentada na literatura, e, estudos recentes da interação do zinco com o ácido fólico, anteriormente considerada danosa em relação ao zinco, não tem confirmado estes resultados.

\section{ESTUDOSDE MINERAISEM DIETASBRASILEIRAS}

Nos trabalhos realizados em nosso laboratório sobre minerais, verificou-se que da composição alimentar da dieta de alguns grupos da nossa população, fazem parte alimentos ricos em fitatos, inibidores da absorção de Fe e Zn; que o consumo de carnes é em sua maioria baixo, fato que causa não apenas um menor aporte de minerais, mas que também tem influência no aproveitamento dos mesmos, e, por serem dietas monótonas, não proporcionam a quantidade recomendada de todos os nutrientes, podendo levar a deficiências. Na Tabela 1, pode-se verificar valores de minerais encontrados em algumas destas dietas (HARADA, 1993; FÁVARO et al., 1997).

Os resultados desta Tabela foram obtidos, a partir da análise química das dietas, preparadas com alimentos adquiridos no comércio local das regiões estudadas e segundo os hábitos normais de processamento domiciliar. Os dados de consumo alimentar foram obtidos por diferentes metodologias, descritas nas Tabelas. Pode-se observar, que em termos quantitativos ocorreu deficiência de cálcio na maioria delas. Os valores de ferro foram variáveis, entretanto, baixos para alguns grupos. Os níveis de zinco estão bem abaixo do recomendado para alguns grupos da população, como por exemplo, idosos e populações de 
menor renda. Os valores de selênio estão baixos ou limítrofes nas dietas de São Paulo e Mato Grosso e mais altos nas de Manaus e Santa Catarina, provavelmente pelo teor de selênio no solo destas regiões. Quanto ao cobre e magnésio as ingestões estão próximas do mínimo recomendado.

Tabela 1. Ingestão média diária de minerais em dietas brasileiras.

\begin{tabular}{lcccccc}
\hline Dieta & $\mathrm{Zn}(\mathrm{mg})$ & $\mathrm{Se}(\mathrm{ug})$ & $\mathrm{Cu}(\mathrm{mg})$ & $\mathrm{Fe}(\mathrm{mg})$ & $\mathrm{Ca}(\mathrm{mg})$ & $\mathrm{Mg}(\mathrm{mg})$ \\
\hline $\begin{array}{l}\text { Dieta I } \\
\text { São Paulo }\end{array}$ & 10,4 & 36,0 & 1,46 & 15,8 & 525 & 313 \\
$\begin{array}{l}\text { Dieta I }{ }^{* *} \\
\text { São Paulo }\end{array}$ & 11,0 & 18,5 & 0,91 & 10,8 & 1069 & - \\
$\begin{array}{l}\text { Dieta III** } \\
\text { São Paulo }\end{array}$ & 3,8 & 38,8 & 0,32 & 5,4 & 444 & - \\
$\begin{array}{l}\text { Manaus } \\
\text { Amazonas }\end{array}$ & 8,7 & 98,0 & 1,13 & 11,2 & 438 & 252 \\
$\begin{array}{l}\text { Santa Catarina I } \\
<\text { renda }\end{array}$ & 5,2 & 55,3 & 0,69 & 6,4 & 287 & 158 \\
$\begin{array}{l}\text { Santa Catarina II } \\
>\text { renda }\end{array}$ & 9,8 & 114,5 & 1,21 & 11,6 & 508 & 122 \\
$\begin{array}{l}\text { Cuiabá } \\
\text { Mato Grosso }\end{array}$ & 9,9 & 60,0 & 1,12 & 12,5 & 356 & 192 \\
\hline
\end{tabular}

Fonte: COZZOLINO et al.

(*) Dieta I: Dieta de um dia, obtida no restaurante universitário, oferecida para alunos e funcionários da USP.

${ }^{(* *)}$ Dieta II: Dados obtidos por registro alimentar, seguidos da elaboração da dieta média em laboratório e posterior análise.

${ }^{(* * *)}$ Dieta III: Análise em duplicata da dieta consumida por idosos em casas de repouso. SP.

Dieta Manaus: Baseada nos dados de SHRIMPTOM \& GIUGLIANO (1979), dieta preparada e analisada.

Dietas de Santa Catarina: Baseadas em levantamentos de dados da Secretaria de Abastecimento da região, preparadas e analisadas. Dieta Cuiabá: Baseada ENDEF (FIBGE,1978), preparada e analisada.

Tabela 2. Contaminantes em dietas brasileiras.

\begin{tabular}{lccccc}
\hline Dieta & $\begin{array}{c}\mathrm{Cd} \\
\text { (ug/dia) }\end{array}$ & $\begin{array}{c}\mathrm{Hg} \\
\text { (ug/dia) }\end{array}$ & $\begin{array}{c}\mathrm{As} \\
\text { (ug/dia) }\end{array}$ & $\begin{array}{c}\mathrm{Sc} \\
(\mathrm{ug} / \mathrm{dia})\end{array}$ & $\begin{array}{c}\mathrm{Rb} \\
(\mathrm{mg} / \mathrm{dia})\end{array}$ \\
\hline São Paulo & $<13,5$ & 8,6 & 19,1 & 1,30 & 5,6 \\
Manaus & $<32,9$ & 39,2 & 16,7 & 0,45 & 6,0 \\
Santa Catarina I & 7,8 & 7,0 & 51,1 & 0,70 & 3,3 \\
Santa Catarina II & 13,8 & 12,7 & 149,0 & 0,80 & 7,5 \\
Mato Grosso & $<33,7$ & 40,2 & 16,6 & 0,64 & 6,0 \\
WHO & $60-70$ & 43 & 50 & - & - \\
\hline
\end{tabular}

Fonte: FÁVARO, et al. (1997)

WHO (1994)

Dieta São Paulo: Obtida por análise da porção em duplicata da dieta servida para estudantes COSEAS/USP.

Dieta Manaus: Baseada nos dados SHRIMPTOM \& GIUGLIANO (1979), dieta preparada e analisada.

Dietas de Santa Catarina: Baseadas em levantamentos de dados da Secretaria de Abastecimento da região, para famílias de menor (I) e maior (II) renda, preparadas e analisadas.

Dieta Cuiabá: Baseada ENDEF (FIBGE, 1978), preparada e analisada.

Por outro lado, também foram determinados alguns elementos considerados tóxicos. Na Tabela 2, pode-se observar que os limites de segurança propostos pela WORLD... (1994) não foram atingidos na maioria dos casos, entretanto, é preocupante para o mercúrio na dieta de Manaus e mercúrio e arsênio nas de Santa Catarina, provávelmente pelo maior consumo de peixe nestas regiões.

No sentido de verificarmos a possibilidade de interações entre minerais nas dietas estudadas, de acordo com o trabalho de COUZY et al. (1993), fizemos um exercício aritmético, Tabela 3, e notadamente não há necessidade de preocupação. Entretanto, continuando nosso exercício, supor que por exemplo, para atender a recomendação atual de ingestão de cálcio, devemos suplementar estas dietas com um adicional de 600 a 700mg/dia, verificaremos que estas relações podem se tornar críticas para a dieta III(idosos), populaçãode risco para esteelemento. Portanto, a suplementação não pode ser isolada, os demais elementos devem ser também avaliados, neste caso ocorreriam prejuízos para o aproveitamento do zinco e do ferro.

Todas as considerações anteriores foram feitas tomando-se como base a quantidade na dieta, 
que como sabemos, não éa quantidade biodisponível. Experimentos conduzidos em nosso laboratório com animais, indicaram uma biodisponibilidade de zinco que variou de 20 a 30\% em dietas da região de São Paulo e da região Nordeste (DANTAS \& COZZOLINO, 1990; PEDROSA \& COZZOLINO,1993).Trabalhosdebiodisponibilidadede selênio demonstraram por outro lado, que o fator limitante nas dietas de São Paulo e de Mato Grosso foi a quantidade do mineral, que levou a um estado de carência nos animais (BOAVENTURA \& COZZOLINO, 1993; CINTRA \& COZZOLINO, 1993). Estudos sobre biodisponibilidade de ferro em dieta de São Paulo, obtiveram valores ao redor 5\% (COLLI, 1988; COLLI \& BARBÉRIO, 1989).

Tabela 3. Relações minerais vs. minerais (mg/mg) em dietas brasileiras.

\begin{tabular}{|c|c|c|c|c|c|}
\hline Dietas & $\mathrm{Ca} / \mathrm{Zn}$ & $\mathrm{Ca} / \mathrm{Fe}$ & $\mathrm{Fe} / \mathrm{Fn}$ & $\mathrm{Zn} / \mathrm{Fe}$ & $\mathrm{Zn} / \mathrm{Cu}$ \\
\hline Dieta I* (SP) & 50,5 & 33,2 & 1,5 & 0,7 & 7,1 \\
\hline Dieta II ${ }^{* *}(\mathrm{SP})$ & 97,2 & 99,0 & 1,0 & 1,0 & 12,1 \\
\hline $\operatorname{Dieta}_{\mathrm{III}}^{* * *}(\mathrm{SP})$ & 117,0 & 82,2 & 1,4 & 0,7 & 11,9 \\
\hline Dieta Manaus-AM & 50,3 & 39,1 & 1,3 & 0,8 & 7,7 \\
\hline $\begin{array}{l}\text { Dieta Santa Catarina } \\
\text { (< Renda) }\end{array}$ & 55,2 & 44,8 & 1,2 & 0,8 & 7,5 \\
\hline $\begin{array}{l}\text { Dieta Santa Catarina } \\
\text { (> Renda) }\end{array}$ & 51,8 & 43,8 & 1,2 & 0,8 & 8,1 \\
\hline \multirow[t]{2}{*}{$\begin{array}{l}\text { Valores } \\
\text { Críticos \# }\end{array}$} & $>200$ & $\begin{array}{c}>150 \text { (Dieta) } \\
>35 \text { (ambos } \\
\text { c/o supl.) }\end{array}$ & $\begin{array}{c}>20 \text { (Dieta) } \\
>2 \text { (sais) } \\
>10 \text { (supl. Fe) }\end{array}$ & $\begin{array}{l}>6(\text { risco }) \\
<2(\text { s/risco })\end{array}$ & $\begin{array}{l}>30 \text { (risco) } \\
<15 \text { (s/risco) }\end{array}$ \\
\hline & $55,3 \otimes$ & 80,0 & 0,7 & 1,5 & 10,0 \\
\hline RDA(1989) & $66,7 \oplus$ & 53,3 & 1,3 & 0,8 & 8,0 \\
\hline
\end{tabular}

\# Segundo : COUZY, et al. (1993).

$\otimes$ Homens $\oplus$ Mulheres

RDA = Recommended Dietary Allowance, (NATIONAL..., 1989)

\section{CONCLUSÕES}

A partir destas reflexões sobre biodisponibilidade de minerais, o que se pode observar é que ainda muitos trabalhos deverão ser realizados até que se possa ter uma definição precisa das necessidades e recomendações, isto é, o quanto um indivíduo necessita ingerir de minerais para estar em condições adequadas de saúde e para prevenção de doenças. Entretanto, os grandes avanços observados nesta direção nos últimos anos, nos estimula a obter dados mais confiáveis, e de alguma forma contribuir para o melhor conhecimento de nossas dietas e da biodisponibilidade de minerais nestas, e ainda, esperamos convencer jovens pesquisadores a ingressarem nesta área de pesquisa, reduzindo desta forma o tempo para que as recomendações de nutrientes sejam realmente a expressão das necessidades dos indivíduos.

\section{REFERÊNCIASBIBLIOGRÁFICAS}

AGGETT, P.J. Severe zinc deficiency. In: MILLS, C.F.(ed). Zinc in human biology. [s.l.] : Spring-Verlag, 1989. p.259-274.

BEATON, G.H. Statistical approaches to establish mineral element recommendations. Journal of Nutrition, Bethesda, v.126,p.2329S-2336S, 1996.

BEUTLER, E. How little we know about the absorption of iron. American Journal of Clinical Nutrition, Bethesda, v.66,p.419-420, 1997.

BOAVENTURA, G.T., COZZOLINO, S.M.F. Selenium bioavailability in the regional urban diet of Mato Grosso, Brazil.Int J Food Sci Nutr, v.43, p.223-229, 1993. 
BREWER,G.J.YUZBASIYAN-GURITAN, V.,DOH-YELL, L. Use of zinc-copper metabolic interactions in the treatment of Wilson's Disease. Journal of the American CollegeofNutrition, New York, v.9, n.5, p.487-591, 1990.

BRODY, T. Nutritional biochemistry. [s.l.] : Academic Press, 1994.660p.

BURTIS, C., ASHWOOD, E.R. Tietz textbook of clinical chemistry. 2.ed. [s.l. : s.n.], 1994, 2326p.

BUZZARD, I.M., SIEVERT,Y.A. Research priorities and recommendations for dietary assessment methodology. American Journal of Clinical Nutrition, Betheda, v.59, p.275S-280S, 1994. (First Internationalon Conference on Dietary Assessment Methods).

CINTRA, R.M.G.C., COZZOLINO, S.M.F. Selenium bioavailability in a regional diet of São Paulo-Brasil.Int J Food Sci Nutr, v.44, n.3, p.167-173, 1993.

COLLI, C. Biodisponibilidade de ferro em dieta regional de São Paulo. São Paulo, 1988. Tese (Doutorado) - Faculdade de Ciências Farmacêuticas da USP, 1988.

COLLI, C., BARBÉRIO, J.C. In vitro estimation of iron bioavailability in São Paulo regional diet. Recent knowledge of iron and folate deficiency in the world. Colloque Insern, v.197, p.345-347, 1989.

COUSINS, R.J. Metal elements and gene expression. Annual Review of Nutrition, Palo Alto, v.14, p.449-469, 1994.

COUZI, F., KEEN, C.,GERSHWIN, M.E.,MARESCHI,J.P. Nutritional implications of the interactions between minerals. Progress in Food and Nutrition Science, Oxford,v.17,p.65-87, 1993.

DANTAS, R.P., COZZOLINO, S.M.F. Biodisponibilidade de zinco em dieta regional de São Paulo. Archivos Latinoamericanos de Nutrición, Guatemala, v.40, n.2, p.221-230, 1990.

FAIRWEATHER-TAIT, S.J. Bioavailability of dietary minerals. Biochemical Society Transactions, Colchester, v.24, n.3, p.775-780, 1996.

FÁVARO, D.I.T., MAIHARA, V.A., ARMELIN, M.J.A., VASCONCELLOS, M.B. COZZOLINO, S.M.F. Determination of $\mathrm{As}, \mathrm{Cd}, \mathrm{Cr}, \mathrm{Cu}, \mathrm{Hg}, \mathrm{Sb}$, and Se concentrations by radiochemical neutron activation analysis in different brazilian regional diets. Journal Radioanalytical Nuclear, Anticles, v.181, n.2, p.385-394, 1994.

FÁVARO,D.I.T., HUI, M.L.T., MAIHARA,V.A.,ARMELIN, M.J.A., VASCONCELLOS, M.B., YUYANA,L.K., BOAVENTURA,G.T.,TRAMONTE,V.L.,COZZOLINO, S.M.F. Determination of some nutrients and toxic elements in different brazilian regional diets by neutron activation analysis. Journal of Trace Elements and
Electrolytes in Health and Disease, Berlin, n.1/2, 1997. (In press).

FUNDAÇÃO INSTITUTO BRASILEIRODE GEOGRAFIA E ESTATÍSTICA (FIBGE). Estudo nacional de despesas familiares: consumo alimentar. Dados preliminares. Tabelas Selecionadas. Rio de Janeiro, 1978. 208p.

GIBSON, R.S. Principles of nutritional assessment. [s.l.] : Oxford University Press, 1990.691p.

GLAHN, R.P., VANCAMPEN, D.R. Iron uptakeis enhanced in caco-2 cell monolayers by cysteine and reduced cysteinyl glycine. Journal of Nutrition, Bethesda, v.127, p.642-647, 1997.

GLEERUP, A., ROSSANDER-HULTHEN, L., GRAMATKOVSKI,E.,HALLBERG, L. Ironabsorption from the whole diet: comparison of the effect of two different distributions of daily calcium intake.American Journal of Clinical Nutrition, Bethesda, v.61, p.97-104, 1995.

GODDARD, W.P., COUPLAND, K., SMITH, J.A., LONG, R.G. Iron uptake by isolated human enterocyte suspensions "in vitro" is dependent on body stores and inhibited by other metal cations. Journal of Nutrition, Bethesda, v.127,p.177-183, 1997.

GUENTHER, P.M., KOTT, P.S., CARRIQUIRY, A.L. Development of an approach for estimating usual nutrient intake distributions at the population level. Journal of Nutrition, Bethesda, v.127, p.1106-1112, 1997.

HALLBERG, L. Food iron absorption. In: COOK, J.D. (ed). Methods in hematology: iron. New York: Churchill and Livingstone, 1980.p.116-193.

HALLBERG, L., HULTEN, L., GRAMATROVSKI, E. Iron absorption from the whole diet in men: how effective is the regulation of iron absorption? American Journal of Clinical Nutrition, Bethesda, v.66, p.347-356, 1997.

HANKIN, J.H., WILKENS, L.R. Development and validation of dietary assessment methods for culturally diverse populations. American Journal of Clinical Nutrition, Bethesda, v.59, p.198S-200S, 1994. Supplement.

HARADA, L.M. Efeito da cimetidina na biodisponibilidade de zinco e cobre em dieta. São Paulo, 1993. Dissertação (Mestrado) - Faculdade de Ciências Farmacêuticas, USP, 1993.

HATHCOCK, J.N. Vitamins and minerals: efficacy and safety. American Journal of Clinical Nutrition, Bethesda, v.66,p.427-437, 1997. 
JACQUES,P.F., SVLSKY,S.L.,SADOWSKI,J.A., PHILIPS, J.C.C., RUSH, D., WILLETT, W.C. Comparison of micronutrient intake measured by a dietary questionnaire and biochemical indicators of micronutrient status. American Journal of Clinical Nutrition, Bethesda, v.57,p.182-189, 1993.

JOHNSON,P.E. New approaches to establishmineral element requirements and recommendations: na introduction. Journal of Nutrition, Bethesda, v.126, p.2309S-2310S, 1996.

KOHLMEIER, L. Gaps in dietary assessment methodology: meal vs. list-based methods. American Journal of Clinical Nutrition, Bethesda, v.59, p.175S-179S, 1994. (Supplement).

LEVANDER, O.A., WHANGER, P.D. Deliberations and evaluations of the approaches, endpoints and paradigms for selenion and iodine dietary recommendations. Journal of Nutrition, Bethesda, v.126, p.2427S-2434S, 1996.

LINDER, M.C.,HAZEGH-AZAM, M. Copperbiochemistry and molecular biology. American Journal of Clinical Nutrition, Bethesda, v.63, p.797S-811S, 1996.

LÖNNERDAL, B. Bioavailability of copper. American Journal of Clinical Nutrition, Bethesda, v.63, p.821S829S, 1996.

LUKASKI, H.C., PENLAND, J.G. Functional changes appropriate for determining mineral element requirements. Journal of Nutrition, Bethesda, v.126, p.2354S-2364S, 1996.

LYNCH, S.R. Interaction of iron with other nutrients. NutritionReviews, New York, v.55,n.4,p.102-110, 1997.

MELLON, F.A, SANDSTRÖM, B. Stableisotopes in human nutrition, inorganic nutrientmetabolism. [s.l.]: Academic Press, 1996. 156p.

NATIONALRESEARCHCOUNCIL(USA). Recommended dietary allowances. 10.ed. Washington DC : National Academy of Science, 1989. p.205-211.

O’BRIEN, J., MORRISSEY,P.A. Metal ion complexationby products of the Maillard reaction. Food Chemistry, Barking, v.58, n.1-2, p.17-27, 1997.

O’DELL, B.L. Endpoints for determining mineral element requirements; na introduction. Journal of Nutrition, Bethesda, v.126, p. 2345S-2353S, 1996.

PEDROSA, L.F.C., COZZOLINO, S.M.F. Efeito da suplementação com ferro na biodisponibilidade dezinco em uma dieta regional do nordeste do Brasil. Revista de Saúde Pública, São Paulo, v.27, n.4,p266-270, 1993.
REDDY, M.B., COOK, J.D. Effect of calcium intake on nonheme-iron absorption from a complete diet.American Journal of Clinical Nutrition, Bethesda, v.65, p.1820$1825,1997$.

SANDSTEAD, H.H. Requirements and toxicity of essential trace elements, illustrated by zinc and copper. American Journal of Clinical Nutrition, Bethesda, v.61, p.621S-624S, 1995. Supplement.

SANDSTROM, B. et al. Oral iron dietary ligands and zinc absorption. Journal of Nutrition, Bethesda, v.115, p.411-414, 1985.

SOUTHGATE, D.A.T. JOHNSON, I., FENWICK, G. R. Nutrient availability: chemical and biological aspects. AFRC Institute of Food Research, Norwich ed., 1989. 404p. (Special Publication no. 72)

SHRIMPTON, R., GIUGLIANO, R. Consumo de alimentose alguns nutrientes em Manaus-Amazonas (1973-4). Acta Amazônica, Manaus, v.9, n.1, p.117-144, 1979.

TOTH, I., BRIDGES, K.R. Ascorbic acid modulates ferritin translation by an aconitase/IRP switch. Blood, Duluth, v.86,p.127a, 1995. Supplement 1.

TUCKER, K. The use of epidemiologic approaches and meta-analysis to determinemineral elementrequirements. Journal of Nutrition, Bethesda, v.126, p.2365S-2372S, 1996.

VANDENBERG, G.J., BEYNEN, A.C. Influence of ascorbic acid supplementation on copper metabolism in rats. British Journal of Nutrition, London, v.68, p.701-715, 1992.

WHITING, S.J., WOOD, R.J. Adverse effects of high calcium diets in humans. Nutrition Reviews, New York, v.55, n.1, p.1-9, 1997.

WOOD, R.J., ZHENG, J.J. High dietary calcium intakes reduce zinc absorption and balance in humans. American Journal of Clinical Nutrition, Bethesda, v.65, p.18031809,1997.

WORLD HEALTH ORGANIZATION. General standard for contaminants and toxins in foods. The Netherlands: Codex Alimentarius Commission. (CX/FAC 95/12).

YARDICK, M.K., KENNEY, M.A., WINTERFELDT, E.A. Iron, copper and zinc status: response to supplementation with zinc or zinc and iron in adult females. American Journal of Clinical Nutrition, Bethesda, v.49, p.145-190, 1989.

YUYAMA, L.K.O., COZZOLINO, S.M.F. Interaçãodezinco e vitamina A em ratos na lactação. Modelo de deficiência experimental.Archivos Latinoamericanos de Nutrición, Guatemala, v.45, n.4, p.305-309, 1995.

Recebido para publicação e aceito em 13 de outubro de 1997. 